\title{
Changes in serum thyroid hormone levels in psychiatric patients treated with second-generation antipsychotics
}

\author{
Jia-Xuan Zhang ${ }^{\circledR 1}{ }^{1}, X^{\prime}$ un $L^{2}$ \\ ${ }^{1}$ Clinical Lab, Zhongshan Third People's Hospital, Zhongshan, China \\ ${ }^{2}$ Paediatric Research Institute of Hunan Province, Hunan Children's Hospital, Changsha, China
}

\begin{abstract}
Introduction: The objective of the study was to evaluate changes in serum thyroid hormone levels in psychiatric patients after second-generation antipsychotic (SGA) treatment, and to determine differences between monotherapy and polytherapy with SGAs, as well as differences between patients with and without a history of antipsychotics.

Material and methods: A total of 71 patients with baseline thyroid hormone levels within the normal reference ranges were included in this retrospectively study.

Results: After SGAs treatment, the serum levels of free triiodothyronine (FT3), free thyroxine (FT4), total triiodothyronine (TT3), and total thyroxine (TT4) significantly decreased, and serum thyroid-stimulating hormone (TSH) levels significantly increased (changes from baseline: FT3: $-0.31 \mathrm{pmol} / \mathrm{L}, \mathrm{p}<0.001$; FT4: $-2.71 \mathrm{pmol} / \mathrm{L}, \mathrm{p}<0.001$; TT3: $-0.05 \mathrm{nmol} / \mathrm{L}, \mathrm{p}=0.024, \mathrm{TT} 4:-12.36 \mathrm{nmol} / \mathrm{L}, \mathrm{p}<0.001$; TSH: $0.46 \mathrm{mIU} / \mathrm{L}$, $\mathrm{p}<0.001)$. One $(1.4 \%)$ patient had TSH levels higher than $7 \mathrm{mIU} / \mathrm{L}$. Changes in serum levels of TSH and TT3 between patients with or without a history of antipsychotic drugs were significantly different (changes from baseline: TSH: $p<0.001$ vs. $p=0.089 ;$ TT3: $p=0.013$ vs. $\mathrm{p}=0.553)$. Changes in serum TSH levels had a moderate positive correlation with the average daily dose of SGAs $(\mathrm{p}=0.007, \mathrm{r}=0.318)$. Conclusions: After SGA treatment, patients seemed to have a trend of hypothyroidism, but the incidence was low. The preliminary results of this study did not show the necessity of regular monitoring of serum thyroid hormone levels in patients with baseline thyroid hormone levels within the normal reference ranges. (Endokrynol Pol 2020; 71 (4): 292-298)
\end{abstract}

Key words: antipsychotics; psychiatric; thyroid; thyroxine; triiodothyronine; thyroid-stimulating hormone; risperidone; olanzapine; clozapine

\section{Introduction}

Second-generation antipsychotics (SGAs), also known as atypical antipsychotics, are the most widely used antipsychotics. Some receptors can be blocked by SGAs, such as dopamine D1, D2, D3, D4 receptors, serotonin $5-\mathrm{HT}_{1 \mathrm{~A}^{\prime}} 5-\mathrm{HT}_{2 \mathrm{~A}}$ receptors, adrenergic $\alpha 1, \alpha 2$ receptors, histamine $\mathrm{H} 1$ receptors, and muscarinic M1 receptors [1]. Some SGAs can block all of these receptors, like clozapine, while most of them can only block some of these receptors [1], among which, dopamine D2 receptors can be blocked by every SGA to varying extents [2].

Dopamine can inhibit the response of thyroid-stimulating hormone (TSH) to thyrotropin-releasing hormone (TRH), and blocking dopamine receptors can cause a significant increase in serum TSH levels [3-5]. Second-generation antipsychotics, as dopamine D2 receptor antagonists, may elevate serum TSH levels, thereby affecting other thyroid hormone levels, such as triiodothyronine (T3) and thyroxine (T4). However, studies on changes in thyroid hormone levels in patients treated with SGAs are limited. Only a few studies focused on SGAs in monotherapy, and the results were inconsistent [6-8].

Conventionally, antipsychotic treatment is initiated with a single SGA, which is defined as monotherapy. When patients do not respond to monotherapy or the response is suboptimal, other SGAs will be added, or the former SGA will be switched to other SGAs, which can be defined as polytherapy. As far as we know, no previous study has reported the comparison of changes in thyroid hormone levels between SGA monotherapy versus polytherapy.

The first aim of this study was to evaluate changes in thyroid hormones in psychiatric patients after SGA treatment. Based on that, the second aim was to determine differences between SGA monotherapy and polytherapy, and the third aim was to determine differences between patients with or without a history of antipsychotics. 


\section{Material and methods}

\section{Patient population}

The Ethics Committee of the Zhongshan Third People's Hospital approved this study. Because this was a retrospective, noninterventional study and all data were collected anonymously, informed consent was not required. The medical records of inpatients at a psychiatric hospital, the Zhongshan Third People's Hospital, from $1^{\text {st }}$ November 2011 to $16^{\text {th }}$ December 2019, were retrospectively reviewed.

Inclusion criteria were as follows: (i) patients aged 18 to 65 years; (ii) without treatment for psychosis or treatment that may affect thyroid function for at least six months prior; (iii) had received SGAs within 24 hours after admission, and continuously received SGA treatment; (iv) and had received SGAs treatment for at least 14 days. Exclusion criteria: (i) not all five baseline thyroid hormone levels, i.e. free T3 (FT3), free T4 (FT4), total T3 (TT3), total T4 (TT4), and TSH, were within the normal reference ranges; (ii) had taken medications known to affect thyroid hormones, such as antithyroid drugs (methimazole = thiamazole, carbimazole, and propylthiouracil), medicines contain iodine, levothyroxine, or lithium during the study period; (iii) pregnant or breastfeeding women.

\section{Variables}

The following data from the medical records of each patient were collected: gender, age at admission, age at disease onset, smoking habit, weight, history of antipsychotic treatment, psychiatric diagnosis, SGA treatment (total dose and duration of use), concomitant medications, and serum thyroid hormone levels (including FT3, FT4, TT3, TT4, and TSH) at baseline and after admission within one year. The first test result of serum thyroid hormone levels with a minimum of 14 days of SGA treatment was taken as the serum thyroid hormone levels at follow-up.

Serum thyroid hormone levels were measured by chemiluminescent enzyme immunoassay using the Access 2 Immunoassay System (Beckman Coulter, Inc., Fullerton, CA, USA). The normal reference ranges of serum thyroid hormone levels were as follows: FT3: 3.28-6.47 pmol/L, FT4: 7.64-16.03 pmol/L, TT3: $1.01-2.48 \mathrm{nmol} / \mathrm{L}$, TT4: $69.97-152.52 \mathrm{nmol} / \mathrm{L}$, and TSH: $0.49-4.91 \mathrm{mIU} / \mathrm{L}$.

Diagnoses were made by psychiatrists according to the $10^{\text {th }}$ revision of the International Classification of Diseases. During hospitalisation, blood samples were drawn between 07:00 and 08:00 before breakfast, for evaluation of serum thyroid hormone levels. According to the Defined Daily Dose (DDD) of the World Health Organisation, the dose of each SGA was converted into olanzapine equivalents [9]. The average daily dose of SGAs was calculated as the total dose divided by the total number of days of SGA treatment. In the subgroup analysis between patients who received SGA monotherapy or polytherapy, the enrolled subjects were divided into two groups: the monotherapy group and the polytherapy group. Here, we referred to monotherapy as consistent use of one SGA, and polytherapy as a combination of more than one SGA, or switch from one SGA to another SGA, with each SGA taken for at least 14 days. Another subgroup analysis was performed between the antipsychotic drug-naïve group $(\mathrm{ADN})$ containing patients with no history of antipsychotics, and the not-ADN group containing patients with a history of antipsychotics.

The concomitant medications were grouped into nine categories: anxiolytics, antidepressants, mood stabilisers, hypnotics, first-generation antipsychotics (FGAs), antiparkinsonian agents, antidementia agents, anticholinergics, and antiadrenergic agents. Information on the use of other medications that were unlikely to affect thyroid function, such as diuretics, laxatives, expectorants, nonsteroidal anti-inflammatories, and antibiotics, was not collected. Information about any medication taken for less than 24 hours was not collected.

\section{Statistical analysis}

Data were reported as number (percentage) for categorical variables, and median (first quartile $=\mathrm{Q} 1$, third quartile $=\mathrm{Q} 3$ ) for continuous variables. The comparison of demographic and clinical characteristics between subgroups was performed using Mann-Whitney U test for continuous variables, and chi-squared test or Fisher's exact test for categorial variables, as appropriate. Wilcoxon matched-pairs rank test was used to compare thyroid hormone levels at baseline and after SGA treatment. Spearman's rank correlation test was used to calculate correlations between serum thyroid hormone levels and the average daily dose of SGAs, as well as the duration of SGA treatment. All tests of hypothesis were two-tailed with a type-I error rate fixed at $5 \%(\alpha=0.05)$. All statistical analyses were performed using IBM SPSS 20.0 software (IBM SPSS, Inc., Chicago, IL, USA).

\section{Results}

Table 1 shows the demographic and clinical characteristics of the subjects, as well as differences in subgroup analysis. A total of 71 subjects were enrolled in the study. A total of eight SGAs were prescribed in different treatment regimens, with a maximum of three SGAs per patient. The most frequently prescribed SGA was risperidone (36 subjects, 50.7\%). More people in the polytherapy group were treated with clozapine and aripiprazole than in the monotherapy group (clozapine: $40.7 \%$ vs. $4.5 \%, \mathrm{p}<0.001$; aripiprazole: $33.3 \%$ vs. $4.5 \%$, $\mathrm{p}=0.004)$. A longer duration treatment of SGAs was observed in the polytherapy group compared with the monotherapy group (median 129 vs. 56 days, $\mathrm{p}<0.001$ ). In the other subgroup analysis, a significant difference was only found in age at psychiatric disorder onset, which was younger in the not-ADN group than in the ADN group (median 27 vs. 37 years, $\mathrm{p}<0.001$ ).

Changes in serum levels of FT3, FT4, TT3, TT4, and TSH from baseline after SGAs treatment are shown in Table 2. Also, comparisons between the monotherapy group and the polytherapy group, as well as the ADN group and the not-ADN group are outlined in this table. After SGA treatment, FT3 levels in one $(1.4 \%)$ patient, FT4 levels in $11(15.5 \%)$ patients, and TT4 levels in three $(4.2 \%)$ patients were all below the normal reference ranges. The TSH level in four (5.6\%) patients were higher than the normal reference range. Among them, only one $(1.4 \%)$ patient had TSH levels higher than $7 \mathrm{mIU} / \mathrm{L}$. No subject developed hypothyroidism.

Spearman rank correlation test results showed a moderate positive correlation between changes in serum TSH levels and the average daily dose of SGAs $(p=0.007, r=0.318)$. No correlation was found between changes in thyroid hormone levels and duration of SGA treatment $(p>0.05)$. 


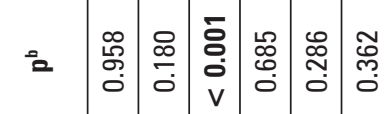

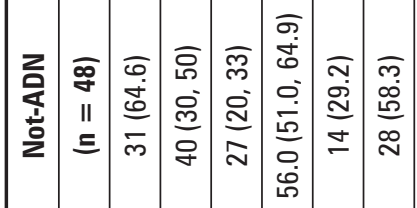

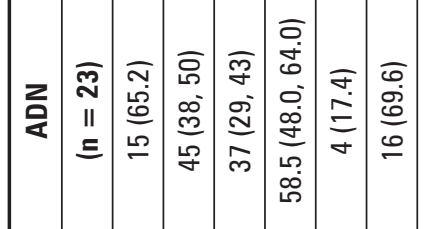

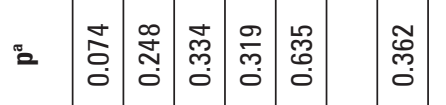

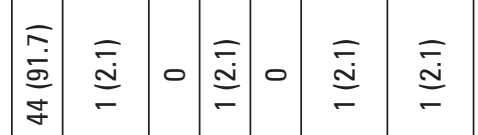

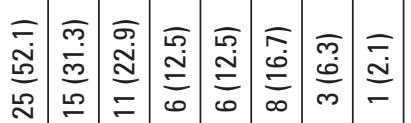

$\frac{\sqrt{2}}{0}$

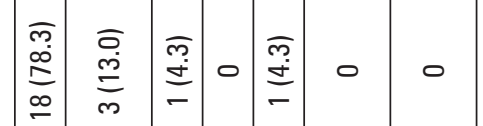

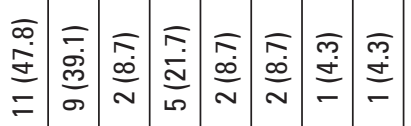

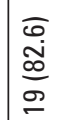

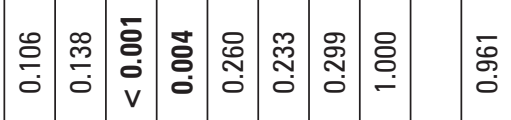

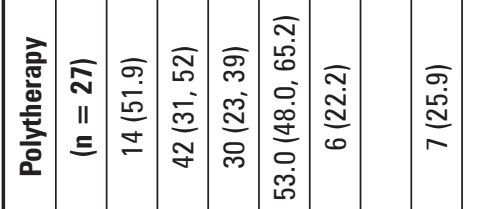

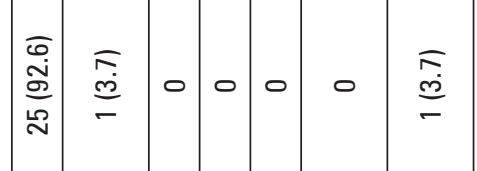

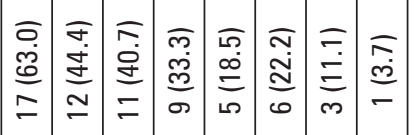

$\stackrel{\widehat{\infty}}{\stackrel{a}{\sim}}$

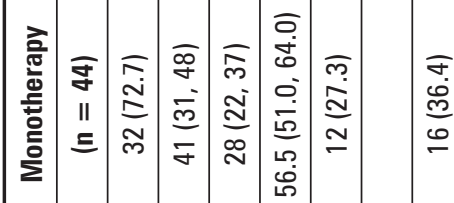

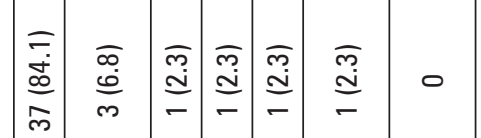

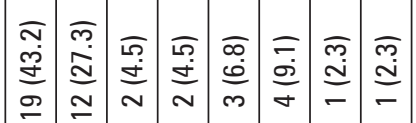

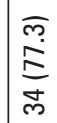

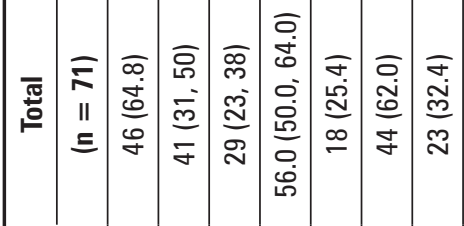

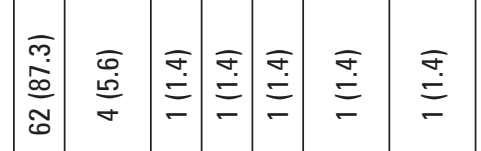

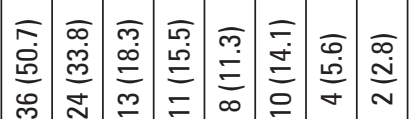

高

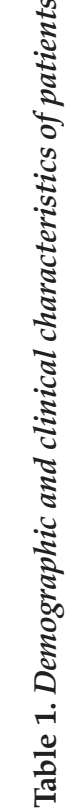
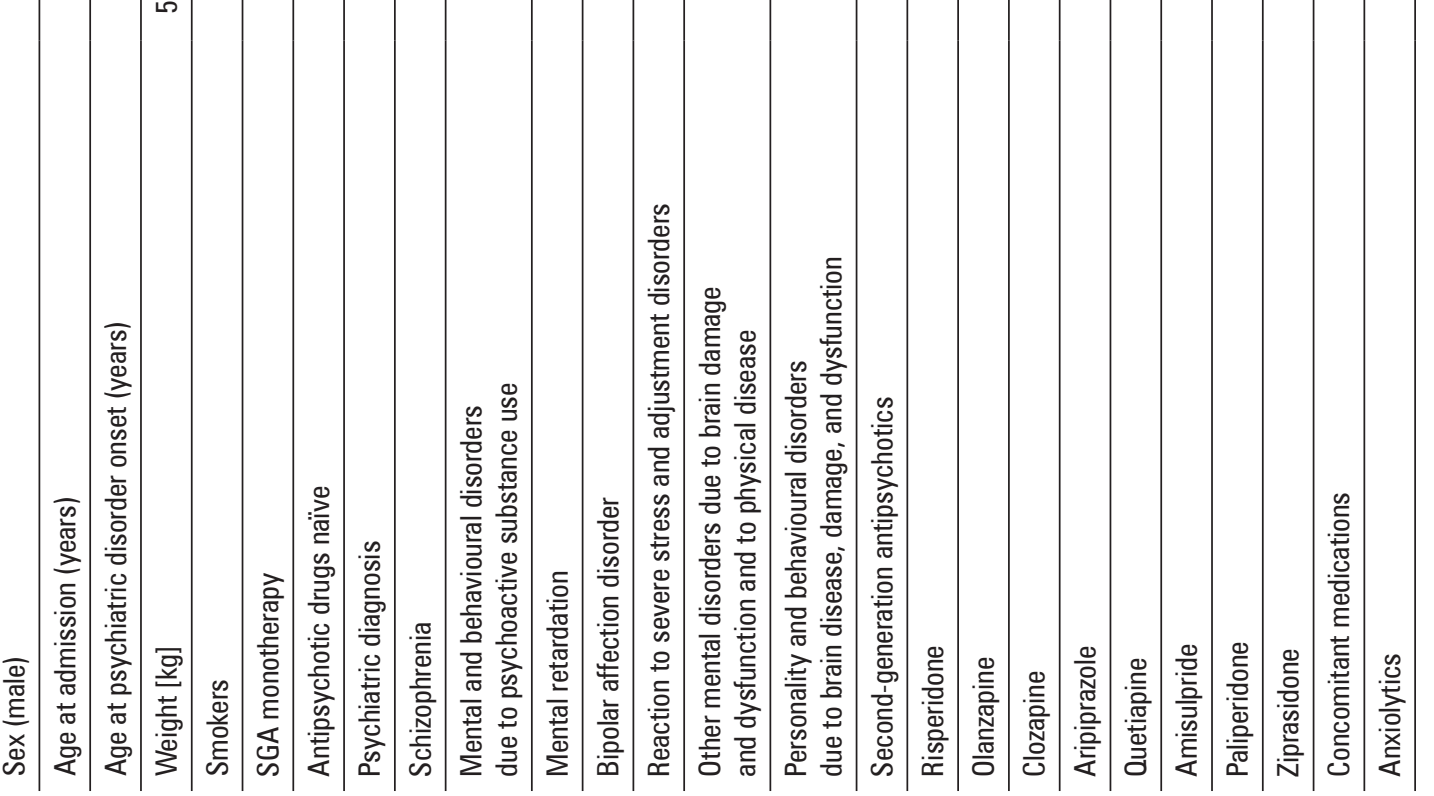


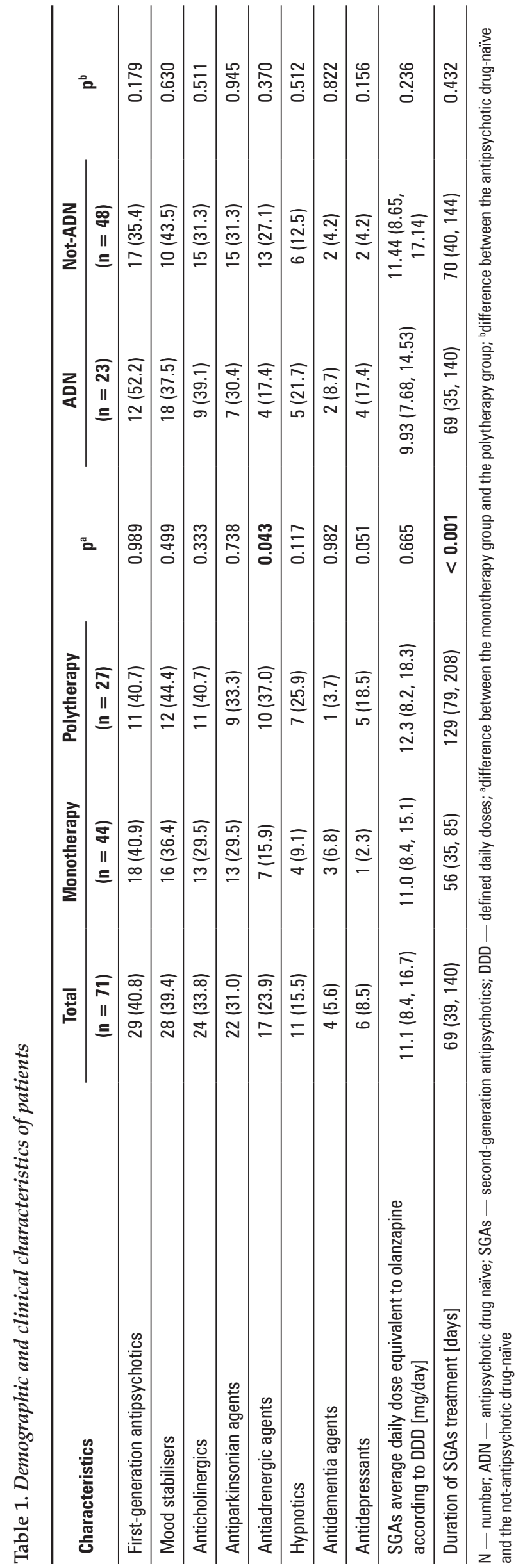

\section{Discussion}

In this study, we analysed changes in serum thyroid hormone levels in psychiatric patients treated with SGAs. Also, we compared differences between SGA monotherapy and polytherapy, as well as differences between patients with or without a history of antipsychotics. Our results showed a significant reduction in the serum levels of FT3, FT4, TT3, and TT4 and a significant increase in serum TSH levels. These results indicate a possible trend of hypothyroidism after SGA treatment. However, only four $(5.6 \%)$ patients developed subclinical hypothyroidism (the normal reference ranges $<\mathrm{TSH}<10 \mathrm{mIU} / \mathrm{L}$ ), without convincing symptoms suggestive of hypothyroidism. Only one (1.4\%) of the four patients had TSH levels high enough (> $7 \mathrm{mIU} / \mathrm{L}$ ) to need treatment for subclinical hypothyroidism [10]. In other words, patients with normal baseline thyroid hormone levels had a low incidence of hypothyroidism after SGA treatment.

Because SGA polytherapy may work when SGA monotherapy fails, changes in thyroid hormone levels may also be different between the two treatment regimens. Surprisingly, subgroup analysis showed no significant difference in changes in serum thyroid hormone levels between SGA monotherapy and polytherapy. Another subgroup analysis found that serum levels of TSH and TT3 were probably more susceptible in patients who had ever taken antipsychotics before than in those who never had (changes from baseline: TSH $\mathrm{p}<0.001$ vs. $\mathrm{p}=0.089$; TT3 $\mathrm{p}=0.013$ vs. $\mathrm{p}=0.553$ ).

Most of the existing studies in this area had been conducted among adolescents and children, with quetiapine being the most frequently studied. Two studies found a decrease in TT4 levels and an increase in TSH levels. One was an eight-week study including 15 psychotic adolescents, which found trends for a decrease in TT4 levels and an increase in TSH levels after quetiapine treatment $(p<0.08)$ [11]. The other was a 12-week study involving 56 adolescents with schizophrenia, which found a significant decrease in TT4 levels ( $p$ 0.001) and a significant increase in TSH levels $(p=0.014)$ after quetiapine treatment [12]. Two other studies had conflicting results. One was a multicentre, double-blind, randomised, placebo-controlled study in adolescents with bipolar depression, which found that extended-release (XR) quetiapine possibly elevated TSH levels compared to placebo (quetiapine XR: $\mathrm{n}=92,4.7 \%$; placebo group: $\mathrm{n}=100,0 \%$ ) but could not significantly change FT4 levels [13]. The other was a six-month follow-up study in psychotic children and adolescents, which found that FT4 decreased after quetiapine treatment $(\mathrm{n}=24$, changes from baseline $-0.21 \pm 0.36 \mathrm{ng} / \mathrm{dL}, \mathrm{p}<0.01)$, but not after olanzapine 
Table 2. Changes in serum of free triiodothyronine (FT3), free thyroxine (FT4), thyroid-stimulating hormone (TSH), total triiodothyronine (TT3), and total thyroxine (TT4) from baseline after second-generation antipsychotics treatment

\begin{tabular}{|c|c|c|c|c|c|c|}
\hline Parameter & $\mathbf{N}$ & Baseline & $\mathbf{p}$ & Changes & $\mathbf{p}^{\mathrm{c}}$ & $\mathbf{p}$ \\
\hline \multicolumn{7}{|l|}{ FT3 [pmol/L] } \\
\hline Total & 71 & $5.08(4.48,5.39)$ & & $-0.31(-0.76,0.21)$ & $<0.001$ & \\
\hline Monotherapy & 44 & $5.16(4.53,5.51)$ & \multirow{2}{*}{$0.594^{\mathrm{a}}$} & $-0.29(-0.80,0.35)$ & 0.027 & \multirow{2}{*}{$0.474^{\mathrm{a}}$} \\
\hline Polytherapy & 27 & $5.05(4.46,5.39)$ & & $-0.37(-0.76,0.08)$ & 0.002 & \\
\hline ADN & 23 & $4.92(4.44,5.35)$ & \multirow{2}{*}{$0.403^{b}$} & $-0.46(-0.95,0.04)$ & 0.004 & \multirow{2}{*}{$0.173^{b}$} \\
\hline N-ADN & 48 & $5.14(4.53,5.56)$ & & $-0.25(-0.74,0.24)$ & 0.021 & \\
\hline \multicolumn{7}{|l|}{ FT4 [pmol/L] } \\
\hline Total & 71 & $11.86(10.54,13.81)$ & & $-2.71(-3.67,-0.75)$ & $<0.001$ & \\
\hline Monotherapy & 44 & $11.87(10.74,13.86)$ & \multirow{2}{*}{$0.356^{a}$} & $-2.83(-3.53,-1.02)$ & $<0.001$ & \multirow{2}{*}{$0.683^{\mathrm{a}}$} \\
\hline Polytherapy & 27 & $11.64(10.25,13.73)$ & & $-2.41(-3.91,-0.60)$ & $<0.001$ & \\
\hline ADN & 23 & $11.84(11.07,13.87)$ & \multirow{2}{*}{$0.731^{b}$} & $-3.04(-4.30,-1.02)$ & $<0.001$ & \multirow{2}{*}{$0.212^{b}$} \\
\hline N-ADN & 48 & $12.00(10.35,13.81)$ & & $-2.66(-3.35,-0.64)$ & $<0.001$ & \\
\hline \multicolumn{7}{|l|}{ TSH [mIU/L] } \\
\hline Total & 71 & $1.40(0.89,2.08)$ & & $0.46(-0.06,1.28)$ & $<0.001$ & \\
\hline Monotherapy & 44 & $1.35(0.86,2.03)$ & \multirow{2}{*}{$0.352^{a}$} & $0.44(-0.03,1.21)$ & 0.001 & \multirow{2}{*}{$0.943^{\mathrm{a}}$} \\
\hline Polytherapy & 27 & $1.44(1.12,2.10)$ & & $0.76(-0.47,1.59)$ & 0.019 & \\
\hline ADN & 23 & $1.76(0.92,2.56)$ & \multirow{2}{*}{$0.253^{b}$} & $0.37(-0.66,1.99)$ & 0.089 & \multirow{2}{*}{$0.754^{b}$} \\
\hline N-ADN & 48 & $1.29(0.88,1.99)$ & & $0.60(-0.03,1.23)$ & $<0.001$ & \\
\hline \multicolumn{7}{|l|}{ TT3 [nmol/L] } \\
\hline Total & 71 & $1.58(1.37,1.77)$ & & $-0.05(-0.30,0.12)$ & 0.024 & \\
\hline Monotherapy & 44 & $1.63(1.38,1.79)$ & \multirow{2}{*}{$0.431^{\mathrm{a}}$} & $-0.05(-0.23,0.13)$ & 0.163 & \multirow{2}{*}{$0.410^{\mathrm{a}}$} \\
\hline Polytherapy & 27 & $1.54(1.36,1.74)$ & & $-0.12(-0.33,0.12)$ & 0.054 & \\
\hline ADN & 23 & $1.60(1.37,1.83)$ & \multirow{2}{*}{$0.849^{b}$} & $-0.01(-0.47,0.13)$ & 0.553 & \multirow{2}{*}{$0.410^{b}$} \\
\hline Not-ADN & 48 & $1.58(1.37,1.77)$ & & $-0.08(-0.28,0.06)$ & 0.013 & \\
\hline \multicolumn{7}{|l|}{ TT4 [nmol/L] } \\
\hline Total & 71 & $123.52(110.19,135.47)$ & & $-12.36(-29.28,3.13)$ & $<0.001$ & \\
\hline Monotherapy & 44 & $124.77(110.92,135.06)$ & \multirow{2}{*}{$0.953^{\mathrm{a}}$} & $-11.43(-30.01,2.91)$ & 0.004 & \multirow{2}{*}{$0.758^{a}$} \\
\hline Polytherapy & 27 & $122.31(110.09,137.32)$ & & $-15.13(-27.61,7.67)$ & 0.046 & \\
\hline ADN & 23 & $121.95(110.05,133.81)$ & \multirow{2}{*}{$0.685^{b}$} & $-20.87(-29.74,2.24)$ & 0.009 & \multirow{2}{*}{$0.892^{b}$} \\
\hline N-ADN & 48 & $126.73(112.95,136.86)$ & & $-10.63(-28.86,5.89)$ & 0.011 & \\
\hline
\end{tabular}

$\mathrm{N}$ - number; ADN — antipsychotic drug naïve; N-ADN — not-antipsychotic drug naïve; adifference between the monotherapy group and the polytherapy group;

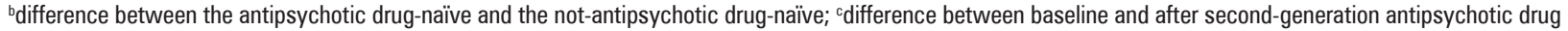
treatment

$(\mathrm{n}=20, \mathrm{p}>0.05)$ or risperidone treatment $(\mathrm{n}=22$, $\mathrm{p}>0.05)$, and TSH did not change significantly after any of the three antipsychotics [14]. The Canadian Alliance for Monitoring Effectiveness and Safety of Antipsychotics in Children guideline group recommended scheduled repeat measurement of TSH levels in patients with normal TSH levels and assessment of FT4 in patients with abnormal TSH levels [15].

Studies in adults are limited, and the results are conflicting. A large cross-sectional study comparing patients with psychotic disorders $(n=1345)$ and healthy controls $(n=989)$ found that patients had lower FT4 levels and higher TSH levels (FT4: 13.70 vs. 14.00 pmol/L, p < 0.001; TSH: 1.92 vs. 1.57 mIE/L, p < 0.001) [8]. Lower FT4 levels were associated with the use of antipsychotics in general $(n=473, p=0.001)$, and quetiapine ( $\mathrm{n}=66, \mathrm{p}=0.003)$, as well as olanzapine ( $\mathrm{n}=148$, $\mathrm{p}=0.018)$ in particular, but were not associated with the use of aripiprazole $(n=64, p=0.402)$ or risperidone ( $\mathrm{n}=43, \mathrm{p}=0.472$ ) [8]. Higher serum TSH levels were not associated with antipsychotics $(p=0.145)$ [8]. No correlations were found between total DDD of antipsychotics and serum FT4 levels ( $p=0.605)$, or serum TSH levels ( $p=0.363$ ) [8]. However, in our study, we found 
a moderate positive correlation between changes in serum TSH levels and the average daily dose of SGAs $(p=0.007, r=0.318)$. Another six-week study on treatment-resistant schizophrenia patients found that TT4 levels significantly decreased after quetiapine treatment ( $\mathrm{n}=10,-2.37 \pm 1.48 \mu \mathrm{g} / \mathrm{dL}, \mathrm{t}=3.93, \mathrm{p}=0.01$ ) but did not change after risperidone treatment $(n=11)$; TSH and FT4 did not change significantly after quetiapine or risperidone treatment [6]. As far as we know, only one study reported changes in the serum levels of FT3, FT4, TT3, TT4, and TSH in the same population, and its results were similar to ours. This was a study in depressed patients with bipolar disorder, which reported that FT3, FT4, and TT4 levels significantly decreased and TSH levels significantly increased after one-month quetiapine treatment, but TT3 did not change significantly $(n=58$; FT4, TT4: $p<0.001$; FT3: $\mathrm{p}=0.011$; TSH: $\mathrm{p}=0.015$; TT3: $\mathrm{p}=0.425)$ [16]. After three-month quetiapine treatment, the serum levels of FT3, FT4, TT3, and TT4 significantly decreased, but TSH did not change significantly $(n=13)$ [16].

Two studies that focused on the risks of hypothyroidism both reported that quetiapine had a higher risk of hypothyroidism than other SGAs $[17,18]$. One was a real-world study in patients with bipolar disorder, which found that the mean four-year risk of hypothyroidism in patients receiving lithium $(\mathrm{n}=3629)$ was $8.78 \%$, and quetiapine $(\mathrm{n}=3798) 8.26 \%$, aripiprazole $(\mathrm{n}=2964) 6.99 \%$, risperidone $(\mathrm{n}=1518) 6.46 \%$, and olanzapine $(n=1230) 6.43 \%$ [17]. The other was a propensity score-adjusted cohort study, which reported a reduction of hypothyroidism in bipolar disorder patients taking olanzapine $(\mathrm{n}=1477$, hazard ratio [HR] $0.48, \mathrm{p}=0.003)$, but not in patients taking quetiapine ( $\mathrm{n}=1376$, HR 0.63, $\mathrm{p}=0.074$ ), compared to lithium $(n=2148)$ [18]. As confirmed by a previous study, lithium can induce hypothyroidism [19-21].

To the best of our knowledge, this is the first study that has compared differences of changes in thyroid hormone levels after treatment between SGAs polytherapy and monotherapy, as well as between patients with or without a history of antipsychotic drugs. In addition, we firstly reported no correlation between changes in thyroid hormone levels and the duration of SGAs treatment ( $p>0.05)$. So far, of all the studies that have reported changes in thyroid hormone levels after SGAs treatment, this is the only study in which all five thyroid hormones of subjects were within the normal reference range at baseline.

Our study had some limitations. Due to the strict inclusion and exclusion criteria, the sample size was small. The eight SGAs were considered as a whole, but the pharmacological effects and usage frequency of each drug were not the same, thus changes in thyroid hormone levels after the monotherapy of each drug might be different. Concomitant medications may also affect thyroid hormones. However, most concomitant medications were not taken chronically. For example, first-generation antipsychotics were usually taken in the first week after admission. Therefore, the effects of concomitant medications on thyroid hormone levels may be limited. Another limitation was the lack of thyroid antibodies assessment and thyroid ultrasound examination, abnormal status of which could affect the results. In fact, patients would undergo thyroid antibodies assessment or/and thyroid ultrasound examination when it was clinically indicated. Those diagnosed with thyroid abnormality, e.g. positive thyroid antibodies or thyroid nodules, would been excluded. Our results should be considered as a preliminary finding about changes in thyroid hormone levels after SGA treatment. Prospective studies with large sample sizes are needed to verify our finding.

\section{Conclusion}

After SGA treatment, psychiatric patients seemed to have a trend of hypothyroidism, but the incidence of hypothyroidism was low. The preliminary results of this study did not show the necessity of regular monitoring of serum thyroid hormone levels in patients with baseline thyroid hormone levels within the normal reference range.

\section{Funding}

This research was supported by the Zhongshan $\mathrm{Mu}-$ nicipal Health Bureau (2018J164).

\section{Acknowledgements}

We would like to thank the Clinical Lab of Zhongshan Third People's Hospital.

\section{Conflicts of interest}

Jia-Xuan Zhang and Xun Li declared no conflict of interest.

\section{Ethics approval}

This study was approved by the Ethics Committees of Zhongshan Third People's Hospital.

\section{Compliance with ethical standards}

All procedures followed were in accordance with the ethical standards of the responsible committee on human experimentation (institutional and national) and with the Helsinki Declaration of 1975, as revised in 2008 (5).

\section{Informed consent}

Because this was a retrospective study, informed consent was not required. 


\section{References}

1. Masana M, Santana N, Artigas F, et al. Dopamine Neurotransmission and Atypical Antipsychotics in Prefrontal Cortex: A Critical Review. Curr Top Med Chem. 2012; 12(21): 2357-2374, doi: 10.2174/1568026128052898 72, indexed in Pubmed: 23279176.

2. Correll CU. From receptor pharmacology to improved outcomes: individualising the selection, dosing, and switching of antipsychotics. Eur Psychiatry. 2020; 25(S2): S12-S21, doi: 10.1016/s0924-9338(10)71701-6, indexed in Pubmed: 20620881.

3. Scanlon MF, Weightman DR, Shale DI, et al. Dopamine is a physiological regulator of thyrotrophin (TSH) secretion in normal man. Clin Endocrinol (Oxf) . 1979; 10(1): 7-15, doi: 10.1111/j.1365-2265.1979.tb03028.x, indexed in Pubmed: 436307.

4. Besses GS, Burrow GN, Spaulding SW, et al. Dopamine infusion acutely inhibits the TSH and prolactin response to TRH. J Clin Endocrinol Metab. . 1975; 41(5): 985-988, doi: 10.1210/jcem-41-5-985, indexed in Pubmed: 810492.

5. Scanlon MF, Mora B, Shale DJ, et al. Evidence for dopaminergic control of thyrotrophin secretion in man. Lancet. 1977; 310(8035): 421-423, doi: 10.1016/s0140-6736(77)90607-9, indexed in Pubmed: 70641.

6. Kelly D, Conley R. Thyroid Function in Treatment-Resistant Schizophrenia Patients Treated With Quetiapine, Risperidone, or Fluphenazine. J Clin Psych. 2005; 66(01): 80-84, doi: 10.4088/jcp.v66n0111, indexed in Pubmed: 15669892.

7. Potkin S, Gharabawi G, Greenspan A, et al. A double-blind comparison of risperidone, quetiapine and placebo in patients with schizophrenia experiencing an acute exacerbation requiring hospitalization. Schizophrenia Res. 2006; 85(1-3): 254-265, doi: 10.1016/j.schres.2006.03.027, indexed in Pubmed: 16797162

8. Vedal T, Steen N, Birkeland K, et al. Free thyroxine and thyroid-stimulating hormone in severe mental disorders: A naturalistic study with focus on antipsychotic medication. J Psych Res. 2018; 106: 74-81, doi: 10.1016/j. jpsychires.2018.09.014, indexed in Pubmed: 30292780.

9. World Health Organization Collaborating Centre for Drug Statistics Methodology. http://www.whocc.no/atcddd/ (Last updated on December 16, 2019.).

10. Ross DS. Subclinical hypothyroidism in nonpregnant adults. . https:// www.uptodate.com/contents/subclinical-hypothyroidismin-nonpregnant-adults (UpToDate. 2019).

11. Shaw J, Lewis J, Pascal S, et al. A Study of Quetiapine: Efficacy and Tolerability in Psychotic Adolescents. J Child Adolesc Psychopharma- col. 2001; 11(4): 415-424, doi: 10.1089/104454601317261591, indexed in Pubmed: 11838824 .

12. Schimmelmann B, Mehler-Wex C, Lambert M, et al. A Prospective 12-Week Study of Quetiapine in Adolescents with Schizophrenia Spectrum Disorders. J Child Adolesc Psychopharmacol. 2007; 17(6): 768-778, doi: 10.1089/cap.2007.0048, indexed in Pubmed: 18315449.

13. Findling R, Pathak S, Earley W, et al. Efficacy and Safety of Extended-Release Quetiapine Fumarate in Youth with Bipolar Depression: An 8 Week, Double-Blind, Placebo-Controlled Trial. J Child Adolesc Psychopharmacol. 2014; 24(6): 325-335, doi: 10.1089/cap.2013.0105, indexed in Pubmed: 24956042.

14. Fraguas D, Merchan-Naranjo J, Laita P, et al. Metabolic and Hormonal Side Effects in Children and Adolescents Treated With Second-Generation Antipsychotics. J Clin Psychiatry. 2008; 69(7): 1166-1175, doi: 10.4088/jcp.v69n0717, indexed in Pubmed: 18588363.

15. Ho J, Panagiotopoulos C, McCrindle B, et al. Management recommendations for metabolic complications associated with second generation antipsychotic use in children and youth. J Can Acad Child Adolesc Psychiatry. 2011; 20(3): 234-241, indexed in Pubmed: 21804854

16. Li C, Lai J, Huang T, et al. Thyroid functions in patients with bipolar disorder and the impact of quetiapine monotherapy: a retrospective, naturalistic study. Neuropsychiatr Dis Treat. 2019; 15: 2285-2290, doi: 10.2147/ndt.s196661, indexed in Pubmed: 31496710

17. Lambert C, Mazurie A, Lauve N, et al. Hypothyroidism risk compared among nine common bipolar disorder therapies in a large US cohort. Bipolar Disord. 2016; 18(3): 247-260, doi: 10.1111/bdi.12391, indexed in Pubmed: 27226264.

18. Hayes J, Marston L, Walters K, et al. Adverse Renal, Endocrine, Hepatic, and Metabolic Events during Maintenance Mood Stabilizer Treatment for Bipolar Disorder: A Population-Based Cohort Study. PLOS Medicine. 2016; 13(8): e1002058, doi: 10.1371/journal.pmed.1002058, indexed in Pubmed: 27483368

19. Bocchetta A, Cocco F, Velluzzi F, et al. Fifteen-year follow-up of thyroid function in lithium patients. J Endocrinol Invest. 2014; 30(5): 363-366, doi: 10.1007/bf03346311, indexed in Pubmed: 17598966.

20. KhalilR, RichaS. Thyroid Adverse Effects of Psychotropic Drugs. Clin Neuropharmacol. 2011; 34(6): 248-255, doi: 10.1097/wnf.0b013e31823429a7, indexed in Pubmed: 21996646.

21. Kibirige D, Luzinda K, Ssekitoleko R. Spectrum of lithium induced thyroid abnormalities: a current perspective. Thyroid Res. 2013; 6(1): 3, doi: 10.1186/1756-6614-6-3, indexed in Pubmed: 23391071. 